\title{
Waveform dependence on signal amplitude in the RHIC H-Jet polarimeter
}

\author{
A.A. Poblaguev
}

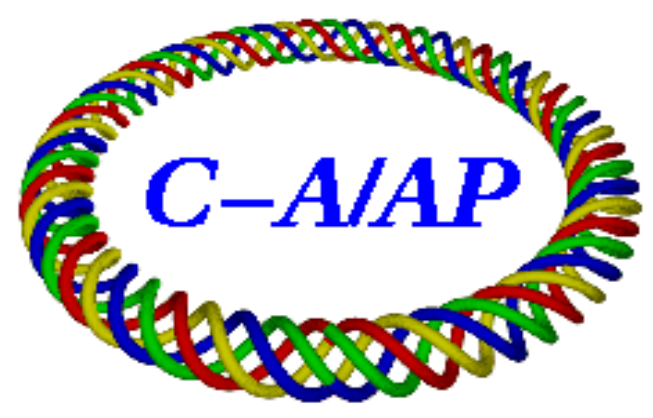

Collider-Accelerator Department Brookhaven National Laboratory

Upton, NY 11973

Notice: This document has been authorized by employees of Brookhaven Science Associates, LLC under Contract No. DE-AC02-98CH10886 with the U.S. Department of Energy. The United States Government retains a nonexclusive, paid-up, irrevocable, world-wide license to publish or reproduce the published form of this document, or allow others to do so, for United States Government purposes. 


\title{
Waveform dependence on signal amplitude in the RHIC H-Jet polarimeter
}

\author{
A.A. Poblaguev
}

February 25, 2014

\begin{abstract}
A simulation of the signal waveform in the H-Jet polarimeter is discussed. The simulation includes a model of charge collection in the silicon detector and a response functions of the H-Jet front end electronics. Results of the simulation are compared with experimental data. It is shown that an analysis of the signal shape may help to suppress background in the H-Jet polarization measurements.
\end{abstract}

\section{Introduction}

The hydrogen jet (H-Jet) polarized target polarimeter [1] is employed to measure the average beam polarization at Relativistic Heavy-Ion Collider (RHIC) polarized proton experiments. For every triggered signal in the silicon $(\mathrm{Si})$ detector, the waveform is measured by effectively $420 \mathrm{MHz}$ 8-bit Wave Form Digitizer (WFD). A typical H-Jet signal waveform $W(t)$ is shown in Fig. 1. Generally it may be parametrized as

$$
W(t)=p+A(t)
$$

where $p$ is signal baseline (pedestal). To identify the elastic $p p$ scattering, the signal amplitude $A_{\max }$ and time $t_{\text {meas }}$ are determined from the analysis of the waveform using the following algorithm.

The waveform baseline $p$ is calculated using the WFD samples with $t<30$ (see Fig. 1) for which $A(t)=0$. After the baseline subtraction, the signal amplitude $A_{\max }$ (relative to the baseline) is counted. At the half-amplitude $A_{1 / 2}=A_{\max } / 2$, the waveform slope is calculated using 6 consecutive waveform points.

$$
A_{\text {slope }}(t)=a+b t, \quad b \simeq d A /\left.d t\right|_{A=A_{\max } / 2}
$$

The measured signal time is defined by a condition $A_{\text {slope }}\left(t_{\text {meas }}\right)=0$ or

$$
t_{\text {meas }}=-a / b
$$

Such a signal time measurement implicitly assumes that the signal's shape is not dependent on the amplitude. Two parameters, $A_{\max }$ and $b$ determined in calculation of the signal time $t_{\text {meas }}$ provide a simple parametrization to monitor the waveform shape:

$$
\Delta t=\frac{A_{\max } / 2}{d A /\left.d t\right|_{A=A_{\max } / 2}}=\frac{A_{\max }}{2 b}
$$




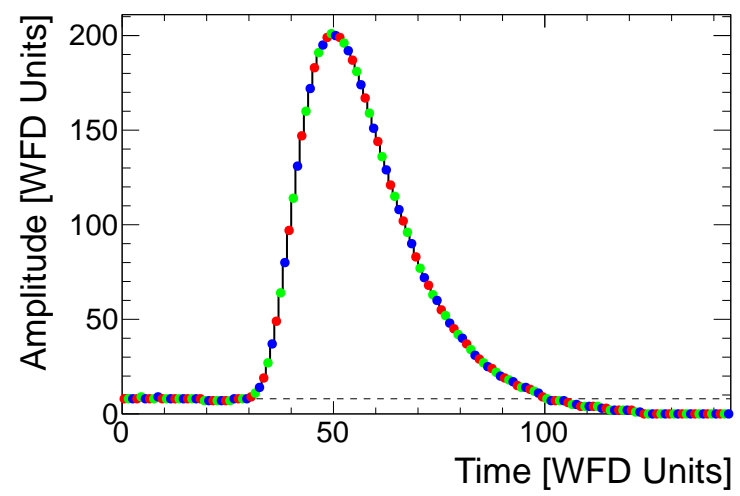

Figure 1: H-Jet signal waveform. The WFD unit is equal to $2.37 \mathrm{~ns}$.

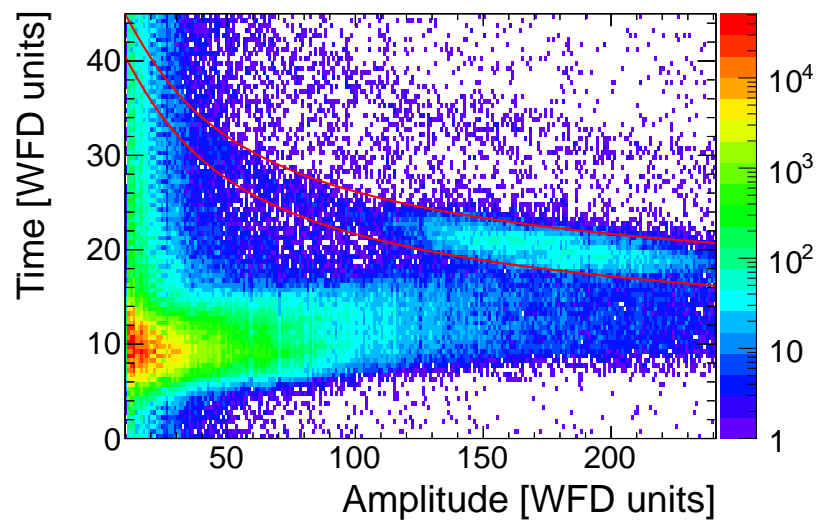

Figure 2: The dependence of measured time on signal amplitude for a single Si strip. The low energy (recoil) proton region is isolated by red lines.

By definition $\Delta t=t_{1 / 2}-t_{\text {meas }}$, where $t_{1 / 2}$ is time corresponding to the half amplitude at raising edge $A\left(t_{1 / 2}\right)=A_{\max } / 2$.

To isolate the recoil proton signal, measured kinetic energy (derived from the $A_{\max }$ ) is compared with its time of flight

$$
\operatorname{tof}\left(A_{\max }\right)=t_{\text {meas }}-t_{0}
$$

The time offset $t_{0}$ is supposed to be determined in a detector calibration.

\section{Time measurement issues in the $\mathrm{H}$-Jet data analysis}

Dependence of the measured time on amplitude for all triggered signals in a single $\mathrm{Si}$ strip is shown on Fig. 2. Two main contributions are low energy recoil protons stopped in the detector and prompt events, which are fast particles penetrating through the 

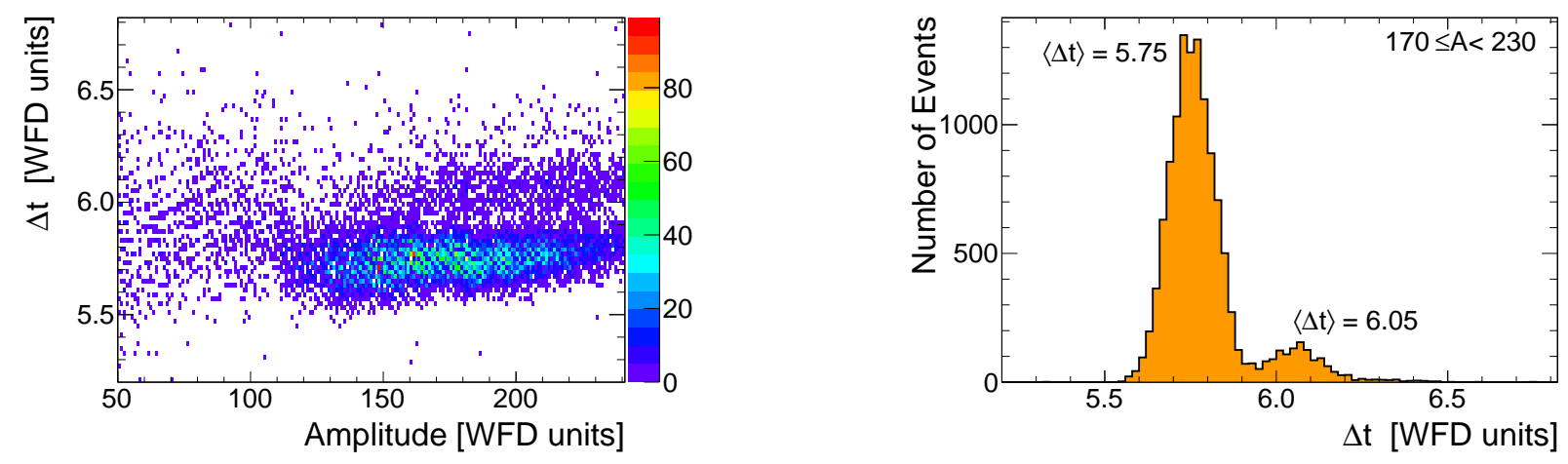

Figure 3: The dependence of the $\Delta t$ on the signal amplitude for events in the recoil proton region in Fig. 2. The $\Delta t$-distribution with additional selection on signal amplitude $170<$ $A<230$ are shown on the right histogram.

detector. The recoil protons are identified by comparison of proton energy $E(A)$ derived from the measured $A$ amplitude and measured signal time $t$ :

$$
E(A)=\frac{M_{p} L^{2}}{2\left(t-t_{0}\right)^{2}}
$$

Here, $M_{p}$ is proton mass and $L$ is the distance to the detector.

Several H-Jet calibration methods were developed [2] to determine the time offset $t_{0}$ and the kinetic energy dependence $E(A)$ on amplitude. Testing of the relationship (6) in the data analysis indicated a dependence of the $t_{0}$ on the signal amplitude $A_{\max }$ $[2]$

$$
t_{0}(A)=\text { const }+(0.50 \pm 0.13) \frac{A-120}{100} \mathrm{~ns}
$$

Since no evidence of incorrect energy calibration was found [2], this result should be interpreted as dependence of measured time on the signal amplitude. For a typical gain $E / A \approx 25 \mathrm{keV} /$ unit, the time offset variates by almost 1 ns within proton energy range (1-5 MeV) used for the polarization measurements. A waveform dependence on signal amplitude might be a possible explanation.

Study of the $\Delta t$ distribution may be considered as a direct test of the uniformity of H-Jet signal waveforms. The dependence of the measured value of $\Delta t$ on the signal amplitude $^{1}$ is shown on Fig. 3. For amplitudes above 160, all events are separated into two distinct groups with $\langle\Delta t\rangle \approx 5.75$ and $\langle\Delta t\rangle \approx 6.05$. The distributions (see Fig. 4) of measured time $t_{\text {meas }}$ relative to the expected time $t_{p}(A)$ for the recoil proton signal with amplitude $A$ allows us to associate the first group with recoil protons and second group with prompts. At a first look it was an unexpected result that the same amplitude signals from recoil protons and prompts have different waveform shapes.

\footnotetext{
${ }^{1}$ for the same events as on Fig. 2
} 

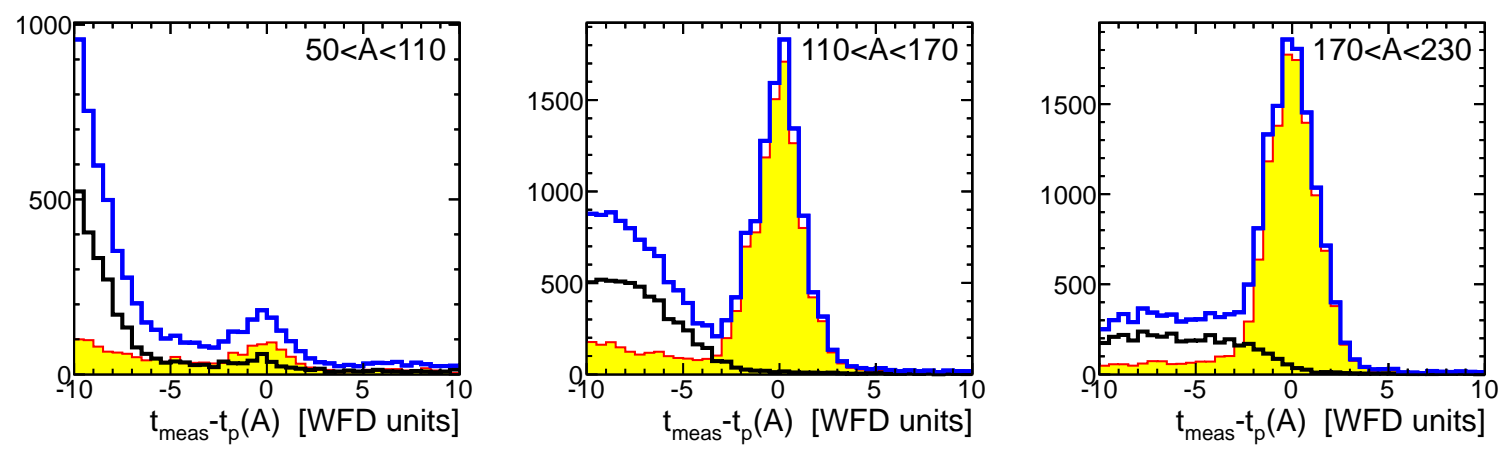

Figure 4: Distributions of the difference between measured time $t_{\text {meas }}$ and expected time for recoil proton signal $t_{p}(A)$ for different ranges of signal amplitudes. Blue lines are for all events, black lines are for the events with $5.95<\Delta t<6.20$ and filled histograms are for the $|\Delta t-5.75|<0.15$ selection.

\section{Simulation of signals in the H-Jet calorimeter}

To understand the phenomena, a simulation of charge collection in the Si strips and the output signal generation was undertaken. The silicon strips were approximated by a detector with parallel plate geometry with uniform field [3]. Assuming very large over-bias, a signal current produced by a single carrier (electron or hole) is proportional to the velocity of the carrier [3]

$$
S_{e, h}(t)=q E_{Q} v_{e, h}=q E_{Q} \mu_{e, h} E=q \frac{\mu_{e, h} V_{b}}{d^{2}}
$$

where $q$ is the carrier charge, $E_{Q}=1 / d$ is the weighting field [3], $\mu$ is the carrier mobility $\left(\mu_{e}=1350 \mathrm{~cm}^{2} V^{-1} \mathrm{~s}^{-1}, \mu_{h}=450 \mathrm{~cm}^{2} V^{-1} \mathrm{~s}^{-1}\right), E=V_{d} / d$ is electric field, and $V_{b}=170 \mathrm{~V}$ is the bias voltage applied across the electrode spacing $d=400 \mu \mathrm{m}$. Since the signal duration is equal to the carrier's drift time to the electrode, the total charge $Q$ induced by electron-hole pair formed at coordinate $x$ from the entrance to the detector

$$
Q=S_{h} \frac{x}{v_{h}}+S_{e} \frac{d-x}{v_{e}}=q
$$

is independent of $x$. So, the integral signal produced by a proton in the detector depends only on the number of formed electron-hole pairs. In other words, it is proportional to the energy deposited in the Si detector.

To simulate the signal waveform we need to know the longitudinal distribution of deposited energy. The number of produced electron-hole pairs is proportional to the Si stopping power $f_{\mathrm{SP}}(E)=d E / d x$ for protons [4]. The dependence of the $d E / d x$ on proton energy is shown on Fig. 5. Using a stopping range definition

$$
\mathcal{L}(E)=\int_{0}^{E} \frac{d \varepsilon}{f_{\mathrm{SP}}(\varepsilon)}
$$

the proton energy $E(x)$ at coordinate $x$ may be calculated as

$$
E(x)=\mathcal{L}^{-1}\left(\mathcal{L}\left(E_{0}\right)-x\right)
$$




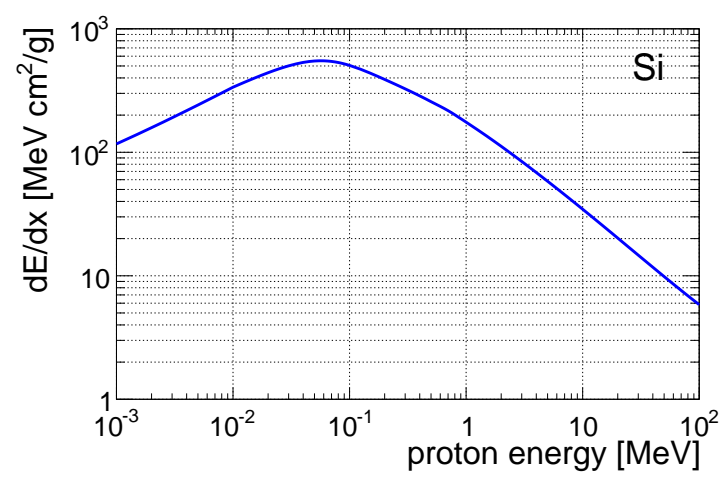

Figure 5: Stopping power of silicon for protons.
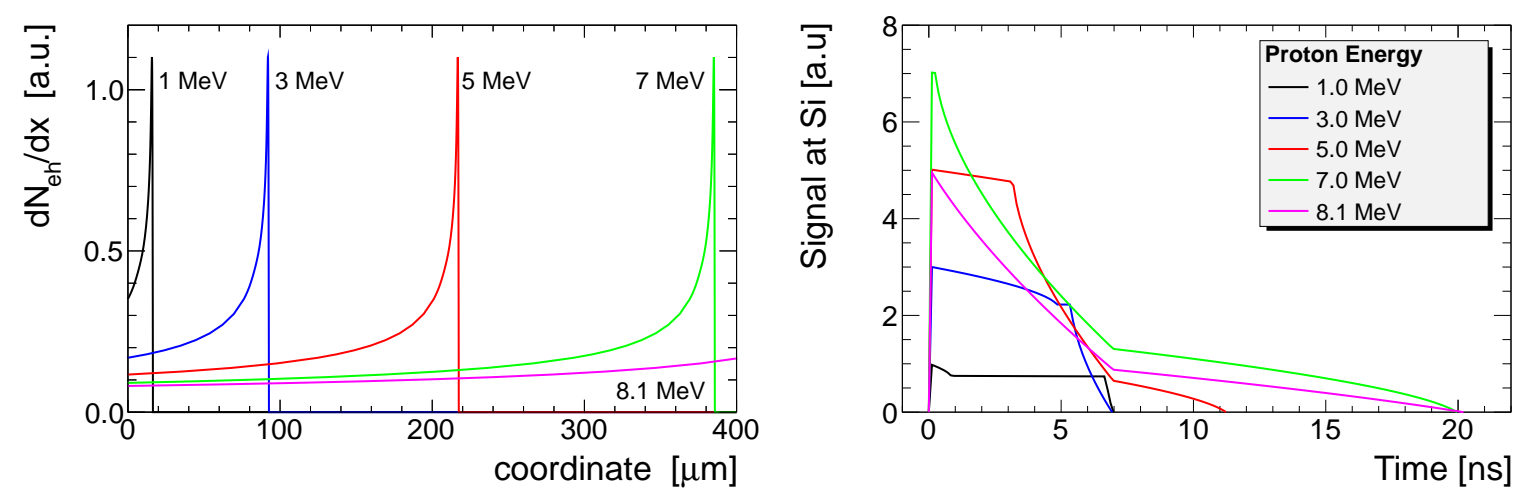

Figure 6: Simulated density of the deposited energy (left) and waveforms at Si strip (right) for several proton energies.

where $E_{0}$ is proton energy at detector entrance, and $\mathcal{L}^{-1}(x)$ is proton energy corresponding to the stopping range $x$.

Results of simulations for several proton energies are shown on Fig. 6. The Si strip dead-layer was not accounted. The energy deposited by a prompt $8.1 \mathrm{MeV}$ proton is approximately equal to $5 \mathrm{MeV}$, the mean energy of elastic protons on Figs. 2 and 3.

One can see a significant dependence of the signal shape on proton energy. However, since the signal $A(t)$ measured by the WFD (see Fig. 1) is a convolution of the signal $S(t)$ at the Si strip and response function $G(t)$ of the electronics

$$
A(t)=\int S(\tilde{t}) G(t-\tilde{t}) d \tilde{t}
$$

a visual dependence of the waveform shape measured in the WFD on the proton kinetic energy is significantly smeared.

In this note we will parametrize the response function as

$$
G(t) \propto t^{n} e^{-t / \tau}
$$



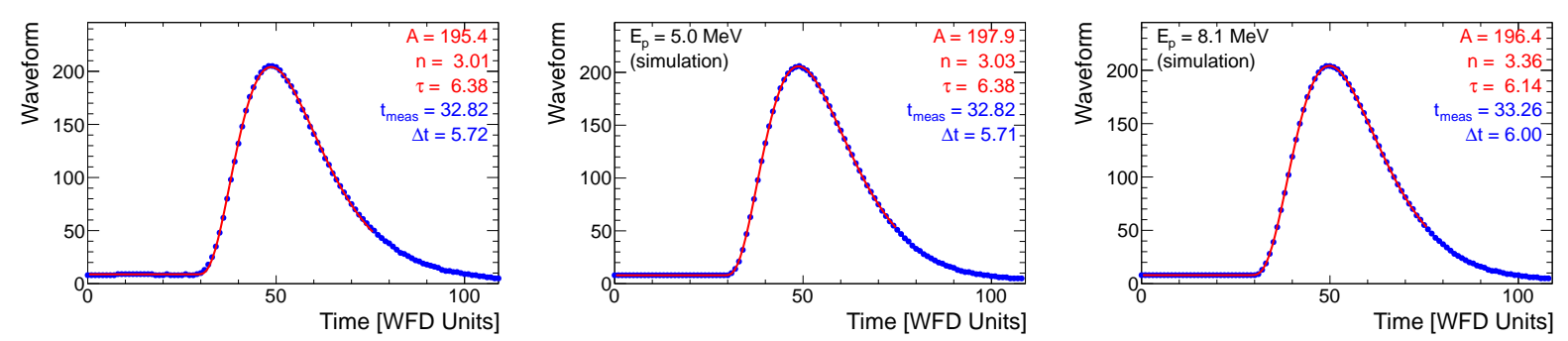

Figure 7: Examples of the waveform processing of the real signal (left) and simulated recoil proton (center) and prompt (right) signals. $t_{\text {meas }}$ and $\Delta t$ are defined in Eqs. (3) and (4), respectively. $A, n$, and $\tau$ are the fit parameters (14). Red lines are the fit functions.

The parameters $n$ and $\tau$ may be evaluated from the fit

$$
W(t)=p+A_{\max } a_{n, \tau}\left(t-t_{s}\right)
$$

of measured waveform. Here $p$ is baseline (pedestal), $A_{\max }$ is signal amplitude, $t_{s}$ is the signal time, and

$$
a_{n, \tau}(t)= \begin{cases}0 & \text { if } t \leq 0 \\ (t / n \tau)^{n} \exp (-t / \tau+n) & \text { if } t>0\end{cases}
$$

is a normalized waveform function. The $a_{n, \tau}(t)$ has maximum equal to 1 at $t=n \tau$. The waveform dependence on signal amplitude is "hidden" in parameters $n$ and $\tau$. The waveform parametrization (7) cannot describe undershoot in the waveform on Fig. 1. For this reason we constrain the fit range by a condition

$$
W(t)>p+0.2 A_{\max }
$$

at the signal back edge as shown on Fig. 7. In addition, to match simulated signal with measured one, the simulated input signal $S_{\text {inp }}$ was modified as

$$
S_{\text {inp }}(t)=\frac{d Q}{d t}=S(t)-\lambda Q(t)
$$

with $\lambda=0.38 \mu$ s. After such a modification the simulated waveform is in a good agreement with a measured one (Fig. 7). In the simulation, the following parametrization of the electronics response function was used:

$$
n=2.74, \quad \tau=6.8 \text { WFD units }
$$

It should be pointed out that parameters (18) which describes the response function of electronics are generally not equal to the parameters $n$ and $\tau$ (see Fig. 7) one determines from the fit of the signal shape measured by WFD.

\section{Simulation of waveform dependence on the proton energy.}

Using a model described above we can simulate the waveform dependence on proton energy. 

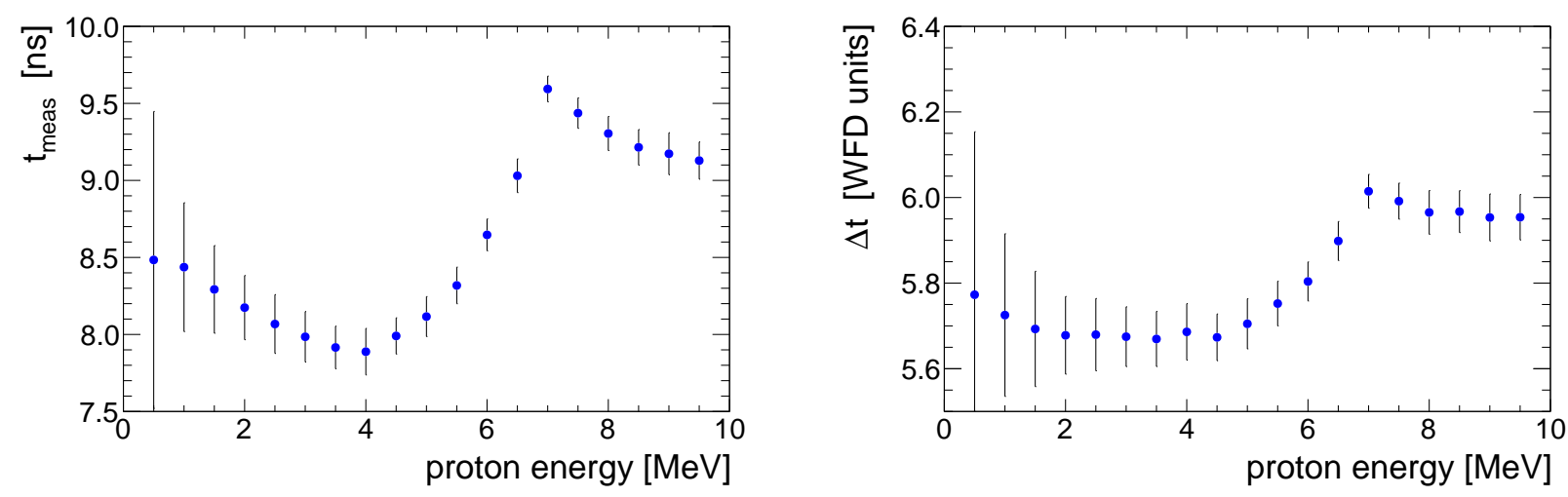

Figure 8: The simulated dependence of measured time $t_{\text {meas }}$ (left) and signal shape parameter $\Delta t$ on proton kinetic energy. Error bars shows the RMS of measured parameters due to the digitization.
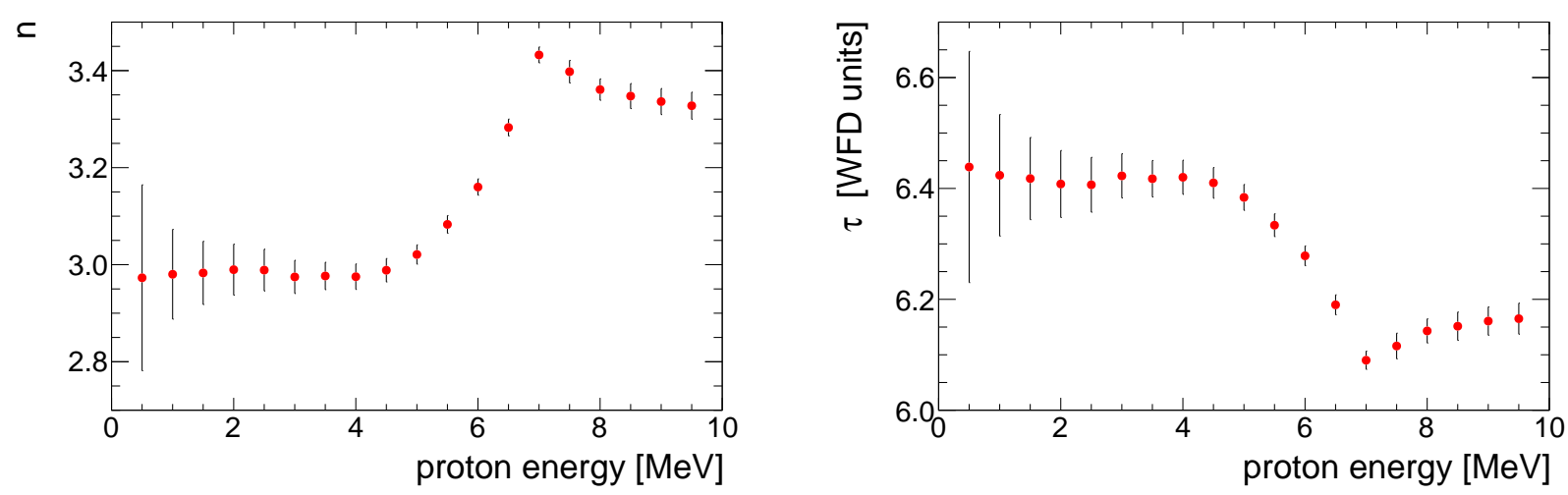

Figure 9: The simulated dependence of waveform fit parameters $n$ and $\tau$ on proton kinetic energy. Error bars shows the RMS of measured parameters due to the digitization.

The simulated dependence of measured time $t_{\text {meas }}$ and normalized front edge slope $\Delta t$ on proton energy are shown on Fig. 8 . For proton energy range 1-5 MeV, there is a variation within $0.5 \mathrm{~ns}$ of measured time depending on the proton energy. However, this dependence differs from the one (7) observed in the H-Jet data analysis. It also should be noted that prompt signals are relatively delayed by about $1 \mathrm{~ns}$.

A simulated dependence of the $\Delta t$ on proton energy matches well the observed difference (see Fig. 3) in waveforms for recoil protons and prompt signals. We can employ this effect for suppression of the prompt signals.

As it follows from Fig. 9, the waveform fit parameters $n$ and $\tau$ may be considered as a promising alternative to the $\Delta t$ for separation of elastic $p p$ and prompt signals.

The simulated dependence of the signal amplitude on the proton kinetic energy is shown on Fig. 10. We may note a good linearity for protons stopped in the detector $(E<7 \mathrm{MeV})$. 


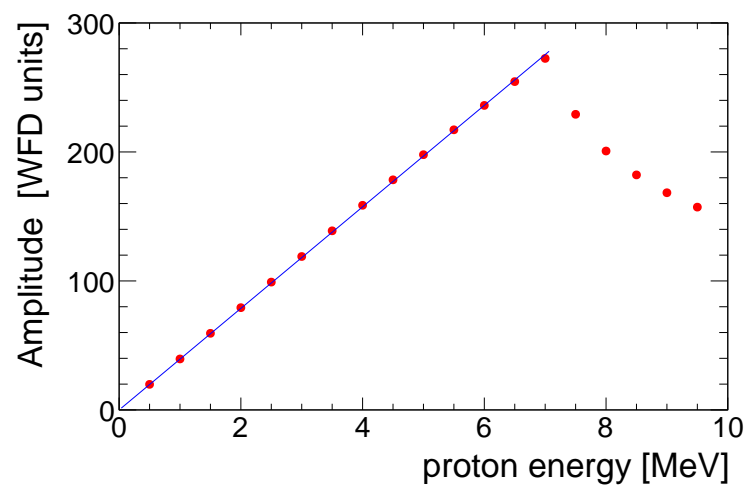

Figure 10: The simulated dependence of signal amplitude (found in the waveform fit) on proton kinetic energy

\section{Summary}

To understand the time measurement issues in the H-Jet data analysis, the simulation of the measured signals was undertaken.

It was shown that waveforms, in a good agreement with observations, are different for the recoil protons and prompt signals. This effect may be employed for background suppression.

Some dependence of measured signal time on proton kinetic energy was found in simulation. However, it can not explain the observed dependence of $t_{0}$ on signal amplitude and even enhance the problem.

\section{Acknowledgments}

I would like to thank Igor Alekseev for stimulating discussions which originated this work.

\section{References}

[1] H. Okada et. al., Phys.Lett. B638 (2006) 450.

I. G. Alekseev et. al., Phys. Rev. D79:094014,2009

[2] A. Poblaguev et. al., The polarized hydrogen jet target measurements at RHIC, presented at XVth International Workshop on Polarized Sources, Targets, and Polarimetry, September 9-13, 2013 Charlottesville, Virginia, USA.

[3] H. Spieler, Semiconductor Detector Systems, Oxford Univ. Press (2005).

[4] http://physics.nist.gov/PhysRefData/Star/Text/PSTAR.html

ICRU (1993). International Commission on Radiation Units and Measurements. ICRU Report 49, Stopping Powers and Ranges for Protons and Alpha Particles.

[5] Hiromi Okada, Measurement of the Analyzing Power $A_{N}$ in pp Elastic Scattering in the CNI Region with a Polarized Atomic Hydrogen Gas Jet Target, Ph.D 
Thesis, Kyoto University, 2006

http://www.bnl.gov/userscenter/Thesis/2007/Okada_Thesis.pdf 